\title{
Solvable Model for Dynamic Mass Transport in Disordered Geophysical Media
}

\author{
M. Marder ${ }^{*}$ and Behzad Eftekhari \\ Department of Physics, The University of Texas at Austin, Austin, Texas 78712, USA \\ Tadeusz W. Patzek \\ Petroleum Engineering Research Center, King Abdullah University of Science and Technology, Thuwal 23955, Saudi Arabia
}

(Received 26 November 2017; revised manuscript received 31 January 2018; published 29 March 2018)

\begin{abstract}
We present an analytically solvable model for transport in geophysical materials on large length and time scales. It describes the flow of gas to a complicated absorbing boundary over long periods of time. We find a solution to this model using Green's function techniques, and apply the solution to three absorbing networks of increasing complexity.
\end{abstract}

DOI: 10.1103/PhysRevLett.120.138302

Introduction.-Transport in disordered media is an enduring physics problem. Much of the work on this topic descends from the original papers on electron localization [1] and percolation [2]. Disordered geophysical materials present special challenges. This is partly because they naturally involve length and time scales too large for direct laboratory experiments. Furthermore, geophysical disorder involves multiscale fractures that impart complex correlations to the transport paths. Ideas from percolation theory have frequently been employed in geophysics [3], but the ideas from electron transport have not.

Here we introduce models that open new possibilities to study transport problems with geophysical characteristics. Like percolation theory they use a lattice where some elements have infinite conductivity. However, the questions we pose are dynamical and best addressed by extending methods developed for electron transport.

We present an example that emerged in the study of gas recovery from hydrofractured shales $[4,5]$. The formations in this case have areas of hundreds of square kilometers and are tens of meters thick. This justifies starting with a twodimensional model. The hydrofracturing process produces a complex network of fractures, propped open with sand of diameter 100 microns or more. Transport within fractures can be treated as an infinitely fast process leading gas to the surface, while everywhere else the transport rate is finite. This is reasonable because outside fractures the transport paths are of nanometer to micron scale. Thus our model describes flow of gas from an infinite two-dimensional plane into a bounded but geometrically complex absorbing boundary. Despite a superficial resemblance to diffusionlimited aggregation (DLA) [6-8] the problem has closest formal relation to the study of electron localization, and is similarly rendered tractable using Green's functions [9-11]. Unlike DLA clusters, our network of absorbers need not be simply connected, or even connected at all. Our main insight was that it is profitable to focus on the time dependence of gas reaching the absorbers. This leads to a tractable model for gas production, showing the possibility of analytical solutions for transport in highly heterogeneous materials on long length and time scales.

Model definition.-We pose the model on a twodimensional square lattice, whose nodes are indexed by $j$. On every node we place a time-dependent density of gas $\rho_{j}(t)$. We denote the state vector of densities by $|\rho(t)\rangle$, and the density at location $j$ by $\rho_{j}(t)=\langle j \mid \rho(t)\rangle$. The problem begins at time $t=0$ with a uniform initial density $\left|\rho_{\mathrm{i}}\right\rangle \equiv|\rho(0)\rangle ;\left\langle j \mid \rho_{\mathrm{i}}\right\rangle=\rho_{j}(0)=1$. Every site is either rock through which gas diffuses or else an absorber that collects all gas reaching it and sends none back. To keep track of absorbers we define $\theta_{j}$ by

$$
\theta_{j}=\left\{\begin{array}{ll}
1 & \text { if } j \text { is unbroken rock } \\
0 & \text { if } j \text { is an absorber }
\end{array} .\right.
$$

Between every pair of nodes $j, j^{\prime}$ there is a link of strength $k_{j j^{\prime}}=k_{j^{\prime} j}$. We restrict ourselves here to nearestneighbor coupling so $k_{j j^{\prime}}$ is 1 if $j$ and $j^{\prime}$ are nearest neighbors and 0 otherwise. The time evolution of $\rho$ is given by

$$
\frac{\partial}{\partial t}|\rho(t)\rangle=\hat{H}|\rho(t)\rangle
$$

where

$$
\left\langle j|\hat{H}| j^{\prime}\right\rangle=\theta_{j}\left[k_{j j^{\prime}}-B \delta_{j j^{\prime}}\right] \theta_{j^{\prime}} ; \quad B \equiv \sum_{j^{\prime}} k_{j j^{\prime}}=4 .
$$

The $\theta$ functions ensure that when gas reaches an absorber it disappears. Note that $\hat{H}$ is real and symmetric, with all off-diagonal entries either 0 or 1 . The Hamiltonian $\hat{H}$ has as many zero eigenvalues as the number of absorbers. If, 
however, one projects down onto the space of all sites $j$ where $\theta_{j}=1$, then these zero eigenvalues disappear. In this space, $\hat{H}$ has a complete set of eigenvectors $|\alpha\rangle$ with eigenvalues $E_{\alpha}$. This observation is not computationally useful when the spectrum is continuous. However, if one has a closed region completely surrounded by absorbers, with a finite number $N_{s}$ of interior lattice points, then the spectrum is discrete, eigenvalues and eigenvectors can be computed explicitly, and the rate $\dot{Q}_{\text {interior }}$ at which gas is produced is

$$
\dot{Q}_{\text {interior }}=-\sum_{\alpha=1}^{N_{s}} E_{\alpha} e^{E_{\alpha} t}\left|\left\langle\alpha \mid \rho_{\mathrm{i}}\right\rangle\right|^{2} .
$$

Green's function solution for continuous spectrum.The exterior region of the absorbing network has an infinite number of sites, the spectrum has a continuous component, and explicit calculation of all eigenvalues is impossible. Obtaining the continuous spectrum requires a different approach. Define the Green function [9] $\hat{G}(E)=$ $(E-\hat{H})^{-1}$ and the unperturbed Green function $\hat{G}_{0}(E)=$ $\left(E-\hat{H}_{0}\right)^{-1}$ for the lattice without any absorbers. The full Hamiltonian is $\hat{H}=\hat{H}_{0}+\hat{H}_{1}$ where

$$
\hat{H}_{1}=-\sum_{j j^{\prime}}|j\rangle\left(k_{j j^{\prime}}-B \delta_{j j^{\prime}}\right)\left(1-\theta_{j} \theta_{j^{\prime}}\right)\left\langle j^{\prime}\right| .
$$

Note that $\hat{H}_{1}$ is nonzero only in the space spanned by absorbers and their nearest neighbors. One can obtain the continuous spectrum from computations in this finitedimensional space. The full Green function can be obtained from the scattering $\hat{T}$ matrix defined by $\hat{G}=\hat{G}_{0}+\hat{G}_{0} \hat{T} \hat{G}_{0}$. To find gas production it turns out one does not need the complete $\hat{T}$ matrix, but just the state vector produced by its action on the initial state $|\mathcal{T}(E)\rangle \equiv \hat{T}(E)\left|\rho_{\mathrm{i}}\right\rangle$. This state vector is determined by the linear system

$$
\left(1-\hat{H}_{1} \hat{G}_{0}(E)\right)|\mathcal{T}(E)\rangle=\hat{H}_{1}\left|\rho_{\mathrm{i}}\right\rangle
$$

and then gas production due to the continuous spectrum of the exterior is provided by

$$
\dot{Q}_{\text {exterior }}=-\int_{-8}^{0} \frac{d \lambda}{\pi \lambda} e^{\lambda t} \operatorname{Im} \lim _{\eta \rightarrow 0}\left\langle\rho_{\mathrm{i}} \mid \mathcal{T}(\lambda-i \eta)\right\rangle .
$$

Because of the limit $\eta \rightarrow 0$, which also applies implicitly to subsequent equations, Eq. (7) includes no contributions from the gas at interior sites captured by Eq. (4). The spectrum of the interior sites is discrete, consists of a finite sum of delta functions, and these vanish in the limiting process. The exterior problem can also have a discrete spectrum [12], missing from this expression, as we discuss later.
Computation of Eq. (6) requires computing matrix elements $\lim _{\eta \rightarrow 0}\left\langle j\left|\hat{G}_{0}(\lambda-i \eta)\right| j^{\prime}\right\rangle$ of the unperturbed lattice Green function. This can be done using recursion relations due to Morita [13], and described in compact form by Berciu [10]. These recursion relations are exponentially unstable, and the instability is particularly severe as $\lambda$ approaches the band edge at $\lambda=0$. One must get very close to the band edge since values as small as $\lambda \approx-10^{-1000}$ are needed for the integral in Eq. (7) to converge. The solution we have adopted is to employ Morita's recursions with high-precision arithmetic. For absorber networks extending across 100 lattice sites, the recursions require from 100 to 5000 places of precision. Once the Green function matrix elements have been obtained, the rest of the computation can be carried out in ordinary double precision.

One absorber.-As a first application of the formalism, consider the case of a single absorber in an infinite square lattice. The vector $\langle j \mid \mathcal{T}\rangle$ has five components. The first corresponds to the absorber at 0 , and the remaining four correspond to the four neighbors of the absorber. Only $\langle 0 \mid \mathcal{T}\rangle$ has a nonzero imaginary part; the remaining values $\langle j \mid \mathcal{T}\rangle=-1$, where $j=1, \ldots, 4$, and

$$
\langle 0 \mid \mathcal{T}(\lambda)\rangle=\lambda+4-\frac{1}{\left\langle 0\left|\hat{G}_{0}(\lambda)\right| 0\right\rangle} .
$$

This leads to total gas production $\dot{Q}=\dot{Q}_{\text {exterior }}$

$$
\dot{Q}=\int_{-\ln 8}^{\infty} \frac{d f}{\pi} e^{-t e^{-f}} \operatorname{Im} \frac{-1}{\left\langle 0\left|\hat{G}_{0}\left(-e^{-f}\right)\right| 0\right\rangle}
$$

where $f=-\ln \lambda$ and for large values of $t$ gives

$$
\dot{Q} \approx \int_{-\ln 8}^{\infty} \frac{d f}{\pi} e^{-t e^{-f}} \frac{1}{\tau_{0}+\tau_{1} f+\tau_{2} f^{2}} ;
$$

where $\tau_{0}=\frac{(\ln 32)^{2}}{4 \pi^{2}}+\frac{1}{4}, \quad \tau_{1}=\frac{\ln 32}{2 \pi^{2}}, \quad \tau_{2}=\frac{1}{4 \pi^{2}}$.

A plot of Eq. (9) appears in Fig. 1.

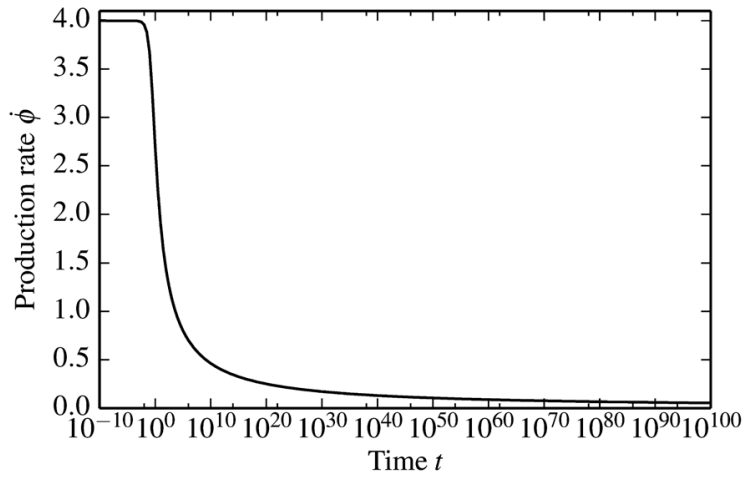

FIG. 1. Production rate of a single absorber over very long time. 


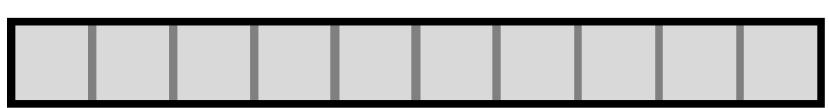

(a)

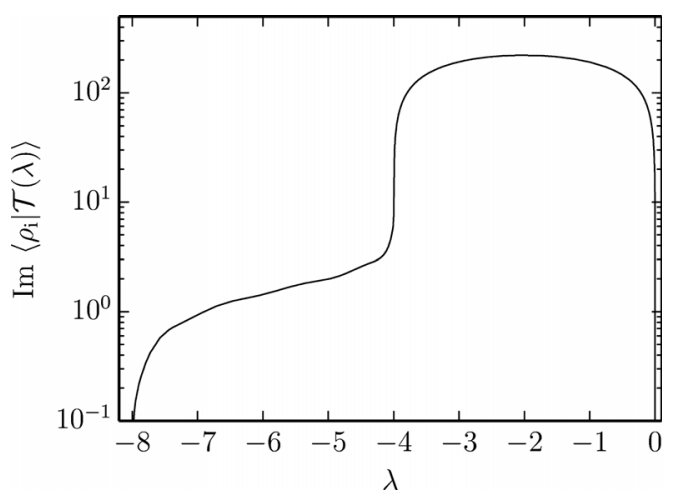

(b)

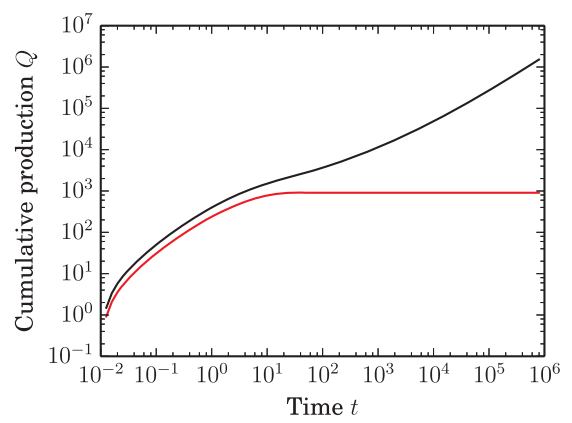

(c)

FIG. 2. Gas production from absorbers that mimic a simplified geometry for hydrofractured wells. (a) Network exhibiting the schematic geometry for hydrofractured horizontal well from [4]. (b) Spectral function from Eq. (7) for network of part (a). (c) Cumulative gas production for network of part (a) (color online). The lower (red) curve gives the production from the interior only.

Absorbing network with ten stages.-As a second example, consider the network pictured in Fig. 2(a). It is 100 units wide, 10 units high, and has 10 internal regions of equal size. The interior problem is of size $810 \times 810$. By counting up the number of absorbing faces exposed to gas, one finds that initial production from the interior must be $\dot{Q}_{\text {interior }}(0)=360$.

The exterior problem leads to a linear system of equations of size $323 \times 323$. Again counting the number of absorbing faces exposed to gas on the outside shows that initial production from the exterior is $\dot{Q}_{\text {exterior }}(0)=224$. The same integer comes from Eq. (7) when $t=0$; this sum rule provides a good check on the accuracy of the computations and shows that the exterior problem has no discrete spectrum in this case. The spectral function $\left\langle\rho_{\mathrm{i}} \mid \mathcal{T}(\lambda)\right\rangle$ appears in Fig. 2(b). The interior and total production appear in Fig. 2(c). The well geometry of Fig. 2(a) is precisely the type of geometry used in Ref. [4] to fit production of thousands of wells.

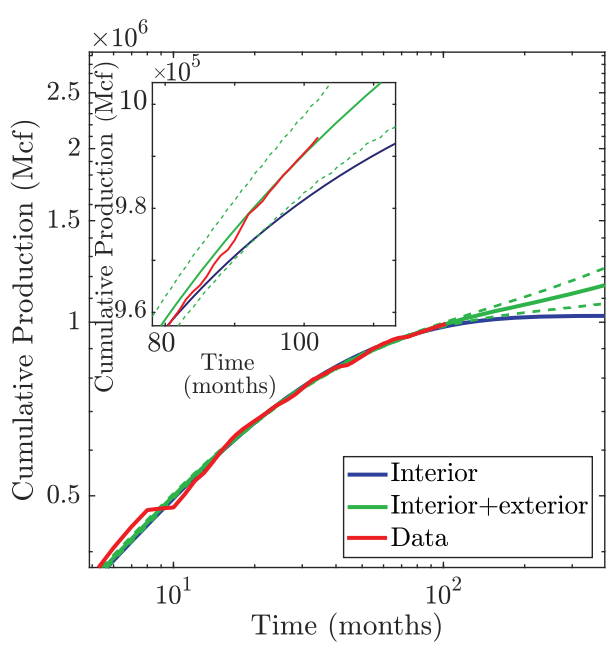

FIG. 3. Well from the Barnett shale that has produced long enough that the contribution from the exterior region is starting to become visible. $\mathrm{Mcf}=1000$ cubic feet. Dashed lines show 95\% confidence interval (color online).

However, only production from the interior of the well was previously considered. Here we see that on sufficiently long time scales, gas arriving from the exterior region leads to a characteristic upturn on a log-log plot. Analysis of well production data to extract this characteristic long-time signature is underway. From the analysis it appears that if one considers a gas-producing well over the course of thirty years, the extra gas produced by exterior flow will contribute extra production on the order of $20 \%$. Figure 3 displays production data from a well for which the longtime behavior including production from the exterior region is just starting to become visible at the ten year mark; this is one of the oldest existing wells.

Complex fracture network.-The essential behavior of the examples presented so far could be examined without much difficulty from a continuum perspective [14] or using finite element programs such as COMSOL. For a final example, depicted in Fig. 4(a), we present a geometrically complex structure produced by 550 intersecting horizontal and vertical cracks with a power-law length distribution motivated by geophysical data [15]. The probability that a fracture has length $l$ is proportional to $l^{-2.2}$. The interior portion, shown with gray scale in the figure, has 3080 sites in light gray that are rock, interspersed by interior absorbers in darker gray. Absorbers forming the exterior of the network are colored in black. The 3080 eigenvalues and eigenvectors of the interior problem needed for Eq. (4) can be computed in seconds on a single processor. The sum rule $\dot{Q}_{\text {interior }}(0)=3264$ provides a check on the accuracy of these computations. The integrand of Eq. (7) has hundreds of narrow spikes that make its computation more difficult although still tractable. Counting up the number of exterior faces on the absorbers gives the sum rule $\dot{Q}_{\text {exterior }}(0)=$ 1556 , but the integral of the continuous spectrum gives only 


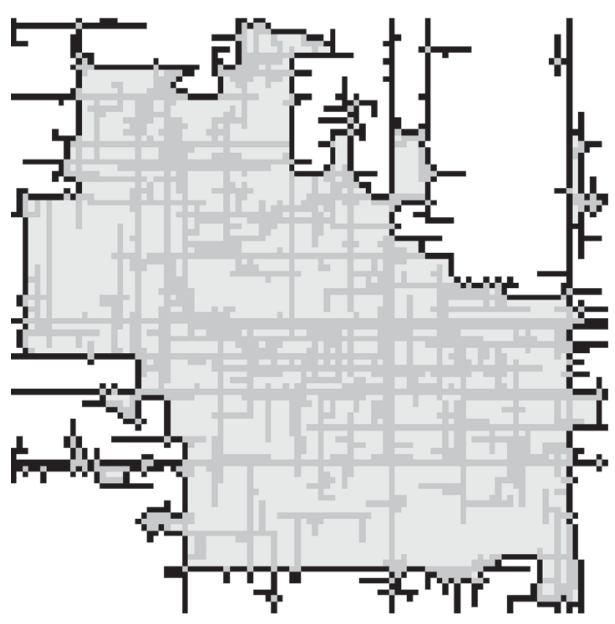

(a)

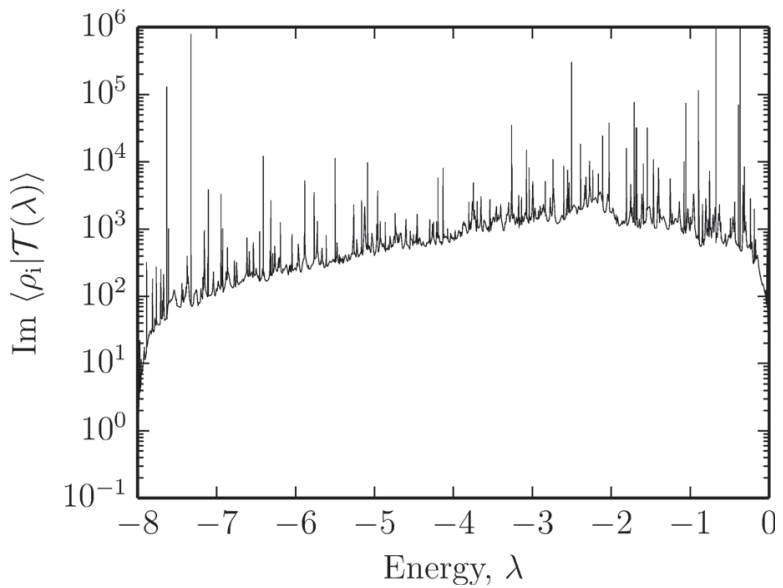

(b)

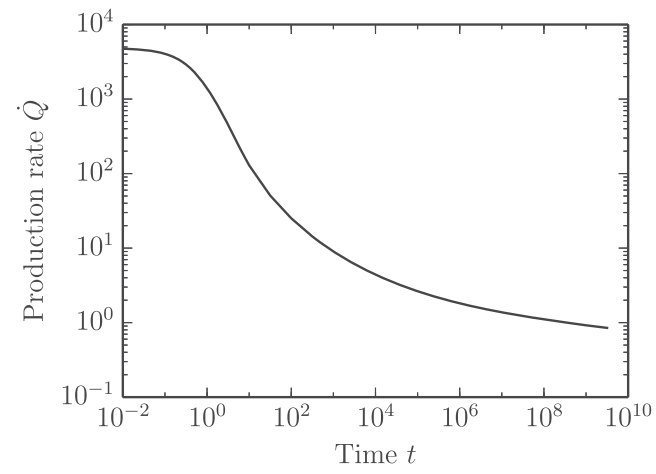

(c)

FIG. 4. Gas absorption into network of criss-crossing fractures. (a) Network of absorbers created by 550 horizontal and vertical cracks. (b) Continuous spectrum for network in part (a). The sharp peaks come from small features on the exterior of the network shielded by extended arms. (c) Total gas production from network in part (a).

1546.53. This is because the external problem has localized eigenfunctions precisely at $\lambda=-4$. Their weight can be determined by taking $\eta=10^{-8}$ in Eq. (7) and performing the integration in a small neighborhood of $\lambda=-4$. The contribution from the exterior localized eigenfunctions is
9.47, and adding this to the integral of the continuous spectrum finally exhausts the sum rule.

For large $f=\ln (-\lambda)$, the continuous spectrum assumes the asymptotic form of Eq. (10) with $\tau_{2}=.02533$, $\tau_{1}=-0.3947, \tau_{0}=1.7877$. This form is all one needs for the very long time behavior. The continuous spectrum appears in Fig. 4(b) and the production rate as a function of time, summing contributions from the discrete and continuous spectra, appears in Fig. 4(c).

This solution illustrates but certainly does not exhaust possible applications of methods from disordered electronic materials to geophysical transport. Possible extensions to complement large-scale simulations are as follows: deduction of fracture geometry from production history, extraction of long-time behavior without reference to fine details, extension to three dimensions, removal of the assumption of complete homogeneity outside fractures, inclusion of thermodynamic properties of real gas, and transport of multiple fluid phases. Improved concepts for geophysical transport are needed if we are to make the most of existing hydrocarbon resources during the inevitable transition to other forms of energy [16].

B. E. acknowledges funding from the King Abdullah University of Science and Technology. We thank David DiCarlo, Carlos Torres-Verdin, and Larry Lake for useful comments as the work was progressing. Qian Niu helpfully pointed out the possibility of localized modes in the continuum.

*marder@mail.utexas.edu

[1] P. W. Anderson, Phys. Rev. 109, 1492 (1958).

[2] S. R. Broadbent and J. M. Hammersley, Math. Proc. Cambridge Philos. Soc. 53, 629 (1957).

[3] A. Hunt, R. Ewing, and B. Ghanbarian, Percolation Theory for Flow in Porous Media (Springer, New York, 2014).

[4] T. Patzek, F. Male, and M. Marder, Proc. Natl. Acad. Sci. U.S.A. 110, 19731 (2013).

[5] D. L. Turcotte, E. Moores, and J. Rundle, Phys. Today 67, 34 (2014).

[6] T. A. Witten, Jr. and L. M. Sander, Phys. Rev. Lett. 47, 1400 (1981).

[7] M. Sahimi, Rev. Mod. Phys. 65, 1393 (1993).

[8] T. C. Halsey, Phys. Today 53, 36 (2000).

[9] E. N. Economou, Green's Functions in Quantum Physics (Springer-Verlag, Berlin, 1983).

[10] M. Berciu and A. M. Cook, Europhys. Lett. 92, 40003 (2010).

[11] G. S. Joyce, J. Phys. A 50, 425001 (2017).

[12] B. Sutherland, Phys. Rev. B 34, 5208 (1986).

[13] T. Morita, J. Phys. A 8, 478 (1975).

[14] H. S. Carslaw and J.C. Jaeger, Conduction of Heat in Solids, 2nd ed. (Oxford, New York, 1959).

[15] B. Eftekhari, Ph.D. thesis, The University of Texas at Austin, 2016.

[16] M. Marder, T. Patzek, and S. W. Tinker, Phys. Today 69, 46 (2016). 